\title{
PC-Based System for Calibration, Reconstruction, Processing, and Visualization of 3D Ultrasound Data Based on a Magnetic-Field Position and Orientation Sensing System
}

\author{
Emad Boctor ${ }^{1,2}$, A. Saad ${ }^{2}$, Dar-Jen Chang ${ }^{2}$, K. Kamel $^{2}$, and A. M. Youssef ${ }^{1}$ \\ ${ }^{1}$ Biomedical Engineering Department, Cairo University Cairo, Egypt \\ eboctor@ieee.org \\ ${ }^{2}$ CECS Department, University of Louisville KY, USA \\ \{eboctor, ashraf, kamel, chang\}@louisville.edu
}

\begin{abstract}
D-ultrasound can become a new, fast, non-radiative, noninvasive, and inexpensive tomographic medical imaging technique with unique advantages for the localization of vessels and tumors in soft tissue (spleen, kidneys, liver, breast etc.). In general, unlike the usual 2Dultrasound, in the 3D-case a complete volume is covered with a whole series of cuts, which would enable a $3 \mathrm{D}$ reconstruction and visualization.
\end{abstract}

In the last two decades, many researchers have attempted to produce systems that would allow the construction and visualization of threedimensional (3-D) images from ultrasound data. There is a general agreement that this development represents a positive step forward in medical imaging, and clinical applications have been suggested in many different areas. However, it is clear that 3-D ultrasound has not yet gained widespread clinical acceptance, and that there are still important problems to solve before it becomes a common tool.

\section{Introduction}

Conventional diagnostic ultrasound imaging is performed with a hand-held probe, which transmits ultrasound pulses into the body and receives the echoes. The magnitude and timing of the echoes are used to create a $2 \mathrm{D}$ gray scale image (B-scan) of a cross-section of the body in the scan plane.

Conventional freehand 3D ultrasound is a multi-stage process [1, 2, 3]. First, the clinician scans the anatomical organ of interest. Next, the ultrasound data is used to construct a 3D voxel array, which can then be visualized by, for example, arbitraryplane slicing. A 3D freehand examination can be broken into three stages: scanning, reconstruction and visualization. 
Before scanning, some sort of position sensor is attached to the probe. This is typically the receiver of an electromagnetic position sensor $[4,5,6,7,8]$, as illustrated in Figure 1, although alternatives include acoustic spark gaps [9], mechanical arms [10] and optical sensors [11, 12]. Measurements from the electromagnetic position sensor are used to determine the positions and orientations of the B-scans with respect to a fixed datum, usually the transmitter of the electromagnetic position sensor.

In the next stage, the set of acquired B-scans and their relative positions are used to fill a regular voxel array. Finally, this voxel array is visualized using, for example, arbitrary-plane slicing, volume rendering or surface rendering (after 3D segmentation).

Freehand systems can be used to obtain arbitrary volumes of data, since the motion of the ultrasound probe is unconstrained. They are also cheap, requiring only existing, conventional ultrasound systems and relatively inexpensive additional components. For these reasons, research into freehand systems is very active.

\subsection{Advantages of 3D Ultrasound System Over Conventional 2D Ultrasound}

There will be many advantages for developing 3D Ultrasound systems in comparison with usual 2D-imaging some of the suggested advantages are:

- The conventional 2D Ultrasound exam is subjective, i.e. it depends on the experience and knowledge of the diagnostician to manipulate the ultrasound transducer, mentally transform the $2 \mathrm{D}$ images into 3D tissue structures and make the diagnosis. While 3D Ultrasound systems will standardize the diagnosis procedure and minimize the dependence on diagnostician experience.

- 2D Ultrasound-guided therapeutic procedures are particularly affected because the process of quantifying and monitoring small changes during the procedure or over the course of time is severely limited by the $2 \mathrm{D}$ restrictions of the conventional exam. This practice is time consuming and inefficient and may lead to incorrect decisions regarding diagnosis and staging, and during surgery. The goal of 3D Ultrasound imaging is to provide an imaging technique that reduces the variability of the conventional technique, and it seems to play an important role in surgical planning.

- It is difficult to localize the thin 2D Ultrasound image plane in the organ, and difficult to reproduce a particular image location at a later time making the conventional $2 \mathrm{D}$ exam a poor modality for quantitative prospective or follow-up studies, while 3D Ultrasound imaging modality will overcome these restrictions.

- In 2D Ultrasound imaging calculating distances and volumes depends on formulas that approximate the body organs to regular geometrical shapes and also depends on the $2 \mathrm{D}$ view that the sonographer calculates the needed distance or volume for a more accurate measurement of distances and volumes.

- Sometimes patient's anatomy or orientation restricts the image angle, resulting in inaccessibility of the optimal image plane necessary for diagnosis. 3D Ultrasound imaging will facilitate an arbitrary selection of a cutting plane and the possibility of reconstructing a 3D-image from the data with several visualization tools. 


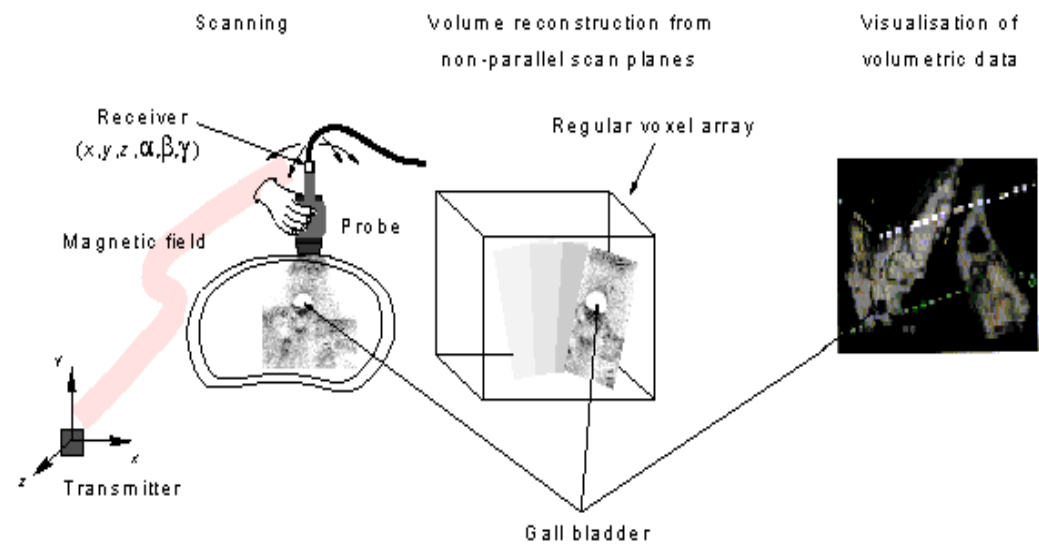

Fig. 1: Conventional freehand 3D ultrasound imaging. This is a multi-stage process, involving scanning, reconstruction and visualization. The figure illustrates an examination of a gall bladder

\subsection{D Ultrasound vs. Other 3D Imaging Modalities: MRI, CT}

Recently, tomographic techniques such as CT, MRI, nuclear medicine, etc., experienced a breakthrough in several domains of diagnostic medical imaging.

3D ultrasound tomography is probably one of the most interesting applications within this area. Several attempts in the past have shown that 3Dultrasound images usually cannot be volume rendered as a 3D-model by using methods known from CT and MRI datasets. The main difficulties originate in:

- The significant amount of noise and speckle in Ultrasound images.

- The regions representing boundaries are not sharp but show a width of several pixels.

- Intensity fluctuations resulting from surface curvature and orientation.

- Self-shadowing of deeper structures.

But in order to gain acceptance, 3-D ultrasound must have clear advantages over the other imaging modalities, e.g. computed x-ray tomography (CT), magnetic resonance imaging (MRI), positron emission tomography (PET) or conventional Bmode and Doppler 2D ultrasound. The main advantages that have been suggested for 3D ultrasound can be grouped into five categories: -

1. Ultrasound is a real time imaging modality, and 3D Ultrasound has the potential for displaying information in near real time too.

2. Extension of ultrasound to 3D provides new images, which would be impossible to visualize otherwise.

3. The reconstruction of 3D Ultrasound by computer potentially brings greater standardization and repeatability to conventional examinations. 


\section{Current State of Knowledge and Different Schools of 3D Ultrasound Research}

Various forms of 3D ultrasound echography have been developed in the past. Among recent examples, several companies, including Tomographic Technologies, Inc. and Acoustic Imaging, Inc., have been working to bring commercial products to market. They acquire 3D echography images by mechanically moving 2D echography slices over periods of seconds to minutes. They then visualize the acquired datasets using volume visualization and other visualization methods.

A real-time 3D ultrasound echography acquisition system is coming close to reality. At Duke University, a prototype, which employs such a parallel processing technique for a real-time 3D echography acquisition system, has been developed[35, 36].

Until now, too many universities and institutes have been working in 3D Ultrasound research area but the most powerful and promising systems exist in the following universities: -

- University of Cambridge, Department of Engineering: This team has developed "Stradx freehand 3D Ultrasound system" [20]. The most important features of this system are: -

Acquisition System: they use electromagnetic position sensors attached to the conventional ultrasound probe to label each B-scan image with its relative position and orientation, Stradx system is characterized in its well-calibrated acquisition system $[15,16]$. Stradx uses a novel, state-of-the-art spatial calibration technique [17]. Visualization Tools: Stradx provides two visualization tools, both of which are available immediately after the data has been acquired, without having to wait for a voxel array to be constructed.

- University of North Carolina at Chapel Hill, Department of computer science: The team at North Carolina has been concentrating on two areas:

I- Real-time 3D ultrasound system: they have been working toward an ultimate 3D ultrasound system, which acquires and displays 3D volume data in real time [21]. Real-time display can be crucial for applications such as cardiac diagnosis, which need to detect certain kinetic features.

II- Augmented reality systems: with see-through head-mounted displays. They explored possibilities for in-place, real-time 3D ultrasound visualization.

- Fraunhofer Institute for Computer Graphics, Germany: The team there developed the first commercial 3D Ultrasound visualization package "In ViVo". It's an interactive system for the fast visualization of 3D Ultrasound datasets on generalpurpose platforms [22]. "In ViVo" supports many visualization tools like:

- Iso-surfaces

- Contour wire frames

- Gradient shading

- Semi-transparent gels

- Max. Intensity projections

- Oblique cuts 


\section{Our Proposed Free Hand 3D Ultrasound System}

We started our research in 3D Ultrasound imaging about 3 years ago. At the first stage we made a survey over nearly all the existing schools working in 3D Ultrasound, we recognize that 3D Ultrasound system is a multi-phase system starts with choosing the hardware suitable for the intended application and ends with the best visualization technique that fits the required target see Figure 3. We found that every research group in this field concentrates on some areas but none of them intends to achieve a whole system for 3D Ultrasound imaging that is suitable to be clinically applied, every school has its own points of power and also has some lag in some phases, for example at UNC Chapel Hill they concentrate to build the whole system for augmented reality environment but they are not concerning with construction and visualization techniques for this system, also at Cambridge University they applied the simplest visualization methods although they have the best calibrated acquisition system for 3D Ultrasound.

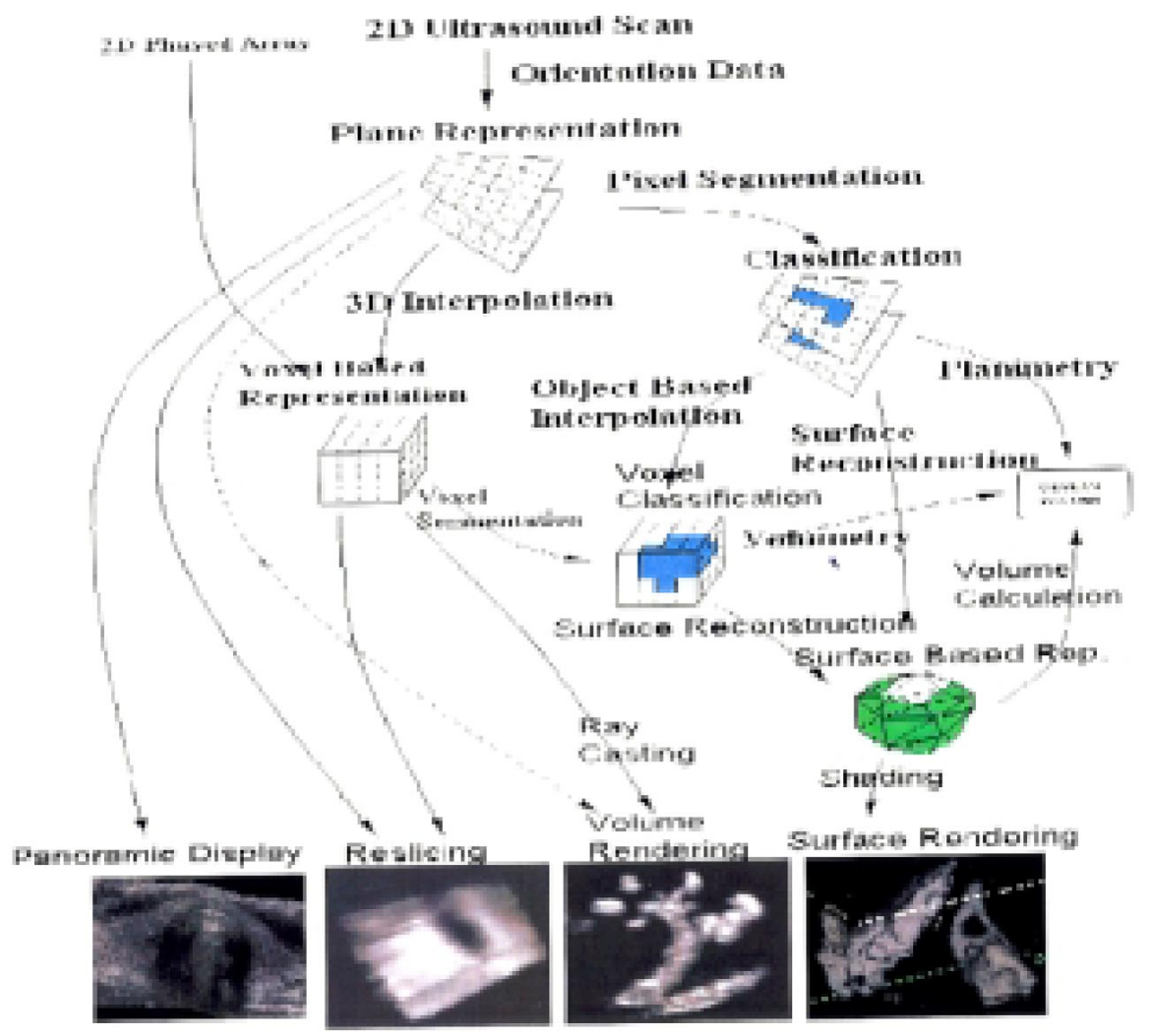

Fig. 3: 3D Ultrasound different systems, Data processing and display

The cost and flexibility of free-hand imaging ensure that it will remain a popular choice for 3D Ultrasound systems. For these reasons, we decided to focus on 
building the infrastructure for a free-hand 3D Ultrasound system. In the next section we will discuss the infrastructure we built in details to indicate the current state of our project.

\subsection{Infrastructure Prototype for 3D Ultrasound System}

Hardware Setup: The following is a list of hardware components that constitute our prototype free hand 3D Ultrasound system:

1- Pentium 200 MHz PC with 128 Mbytes RAM, 4 GBytes SCSI Hard Disk.

2- An electromagnetic position tracker "miniBIRD", see Figure 4, that is a six degree of freedom measuring device that is used to measure the position and orientation of a small receiver with respect to a transmitter.

3 - A digitizer card "Matrox Card" which is real time frame grabber capable of virtually capturing 25 frames/sec.

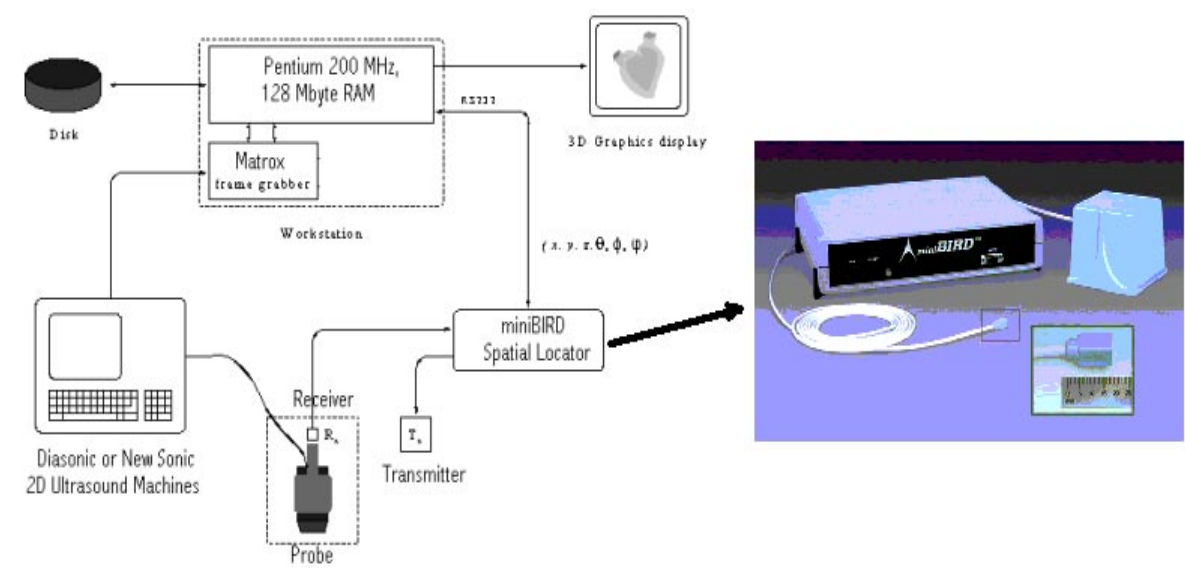

Fig. 4: a system block diagram (left) and miniBIRD electromagnetic position tracker (right)

Operating Platform: We developed our system under "Windows NT" platform, which seems to be a promising choice for the future to develop a system working on a PC under Windows Operating System.

Acquisition System: The first step in the project was to implement a real time acquisition system to capture a set of randomly oriented images guided by their orientation data acquired from the spatial locator. To achieve these goals we followed the next steps:

1- we manufactured a plastic holder that fits over the probe of the ultrasound machine and has a holding position for the receiver of the spatial locator. Some precautions had to be taken as:

-The material of the holder was chosen so that it would not affect the performance of the spatial locator i.e. it should contain no conducting materials. 
- The design should not obstacle the free hand movement of the ultrasound probe as the physician was used before.

- The local axes of the receiver should not be in a special orientation with the local axis of the 2D ultrasound image like a perpendicular or parallel case, this condition is necessary for proper calibration of the spatial locator as will be indicated later.

- When removing the holder and reinstalling it again over the probe it should stay in the same location, this condition is also necessary for proper calibration of the spatial locator as will be indicated later.

2 - A key requirement of all free-hand imaging systems is the calibration of the spatial locator, there are two sources of errors in the readings of the electromagnetic tracker that should be taken into account to improve the registration of the free hand 2D slices.

- The first error in the readings comes from the effect of the proximity of metallic objects and induced noise from power cables exist in the ultrasound clinic's environment, we applied a look-up table technique to calibrate both position and orientation readings.

- The second error originates from the fact that the readings representing the position and orientation of the receiver's center relative to the center of the transmitter (reference point) are not the actual readings that we need, which are the readings representing the position and orientation of the corner of the B-scans relative to the center of the transmitter.

A typical calibration process, which often needs repeating every time a sensor is mounted on a probe, takes several hours for a skilled technician to perform. At Cambridge University, they presented a novel calibration technique which can be performed in a few minutes with a very good accuracy, but they invent a special phantom for this purpose which will add cost to the system and would not be available everywhere. We applied a novel technique to calibrate the spatial locator in a few minutes with a very good accuracy, but we used a very popular phantom for that purpose, which is the "AIUM Tissue Cyst Phantom".

Our technique is based on imaging the phantom from several orientations then entering this images into a program that automatically detect the coordinates of the phantom reflectors, this program was implemented by the authors in a previous project concerning calibration of ultrasound machines $[18,19]$.

3 - After performing the spatial locator calibration, we implemented the acquisition phase of the program that interfaces the digitizer card and the locator (via the serial interface) to the PC. A time synchronization process has been developed to achieve a minimal delay between the acquired 2D- ultrasound images and the associated orientation data.

Compounding and Reconstruction Techniques: The acquired B-images are randomly oriented so we applied a compounding technique that fits the images into an empty volume of voxels, the resolution of the voxel dataset could be changed upon user interface, then we applied different reconstruction techniques that translate the irregular voxel volume into a regular one, that is suitable for visualization algorithms. The reconstruction algorithms, we chose, have the following properties: 
1- they are local so that their computational cost can be bounded,

2- they produce approximations (not interpolations) so that the noise in samples can be suppressed,

3- they produce smooth approximation results, so that the visualization results are also smooth without noisy artifacts,

4- they are insensitive to the order in which the samples arrive, so that the reconstruction results of identical objects are identical regardless of different paths of sweep upon acquisition.

Image Processing Library: Due to the speckle nature of ultrasound images, the visualization techniques that are valid for CT and MRI cannot be directly applied to 3D Ultrasound datasets. Image preprocessing algorithms should be applied first to the reconstructed datasets before visualization. While implementing this phase we put in our mind some goals to achieve:

- The main target of applying image processing routines is to suppress noise and speckle in the volume in conjunction with preserving the edges of the target organ.

- The applied algorithms should be very fast to achieve the global requirement of implementing an interactive system suitable for routine clinical times.

- These algorithms should not alter the dimensions and shape of the organs so that a quantitative analysis could be performed later on the volume.

- These algorithms should be applied to 3D volumes, not repeatedly applied to a set of $2 \mathrm{D}$ images, so that continuity is achieved in all the three dimensions.

Visualization Techniques: We have implemented some visualization techniques as follows:

- We have implemented "Z shading" technique to visualize iso-valued surfaces after performing filtering algorithms necessary for depicting meaningful isovalued surfaces from ultrasound data. Figure 6 shows a rendered scene using "Z shading" for the gall bladder of one of the authors. The main drawback of this method is its limitation for imaging particular structures.

- After that we took the direction of "Volume Rendering", We implemented a Volume Rendering pipeline using "Voxelator Library" which is a prototype volume rendering software as an extension to the OpenGL library. Figure 6 shows a screen of a rendered image by Voxelator library. The main drawback of using OpenGL library for volume rendering is the time consumed to update the rendered scene.

- Finally we have implemented a fast volume rendering technique, developed by Philippe Lacroute at Stanford University. This technique uses a Shear-Warp Factorization of the viewing transformation to accelerate the rendering process. Figure 6 shows a rendered scene for the hepatic veins belonging to one of the authors using this technique. 

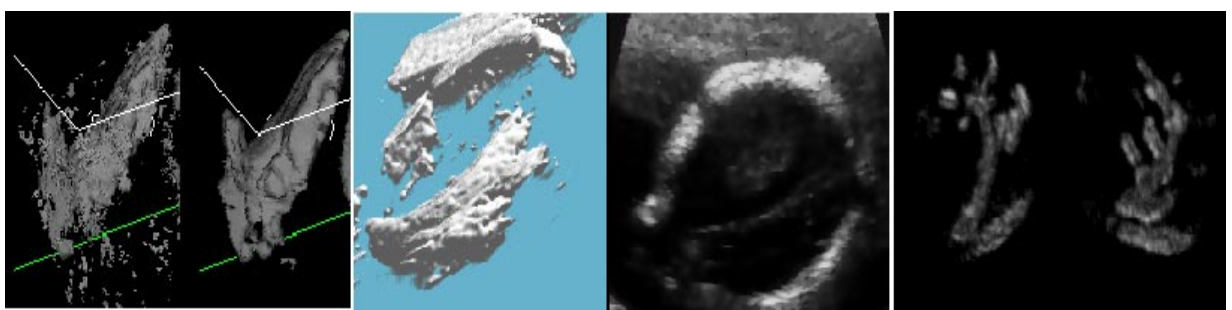

a b c d e

$\mathbf{f}$

Fig. 6: From the left: $\mathrm{Z}$ shading using iso-surfaces for the Gall Bladder of One of the authors a) Without Image Processing. b) With Image Processing. c) Volume rendered scene for the forehead part of a fetus using OpenGL Library. d) A cross section of the forehead of the fetus. e) and f) Two different views of volume rendered Hepatic veins of the author Using Fast Volume Rendering Algorithm.

\section{Conclusion}

In this paper, we have developed a prototype free-hand 3D ultrasound system that is capable of acquiring, constructing, and visualizing 3D ultrasound data sets. The main goal was to achieve high quality rendered images for anatomical structures within a reasonable time compared to the conventional ultrasound diagnostic time. The future research directions of the authors will be targeted on extracting useful quantitative information from the acquired 3D data, which could include 3D segmentation techniques and voxel classification methods.

\section{References}

[1] J. Deng, J. E. Gardener, C. H. Rodeck, and W. R. Lees. Fetal echocardiography in 3dimensions and 4-dimensions. Ultrasound in Medicine and Biology, 22(8):979--986, 1996.

[2] R. N. Rankin, A. Fenster, D. Downey, P. L. Munk, M. F. Levin, and A. D. Vellet. Threedimensional sonographic reconstruction: techniques and diagnostic applications. American J. of Roentgenology, 161(4):695-702, 1993.

[3] H. Steiner, A. Staudach, D. Spitzer, and H. Schaffer. Three-dimensional ultrasound in obstetrics and gynaecology: technique, possibilities and limitations. Human Reproduction, 9(9):1773--1778, 1994.

[4] C. Barry, C. Allot, N. John, P. M. Mellor, P. Arundel, D. Thomson, and J. C. Waterton. Three-dimensional freehand ultrasound: Image reconstruction and volume analysis. Ultrasound in Medicine and Biology, 23(8):1209--1224, 1997.

[5] P. R. Detmer, G. Bashein, T. Hodges, K. W. Beach, E. P. Filer, D. H. Burns, and D.E. Strandness Jr. 3D ultrasonic image feature localization based on magnetic scan- head tracking: in vitro calibration and validation. Ultrasound in Medicine and Biology, 20(9):923--936, 1994. [6] S. W. Hughes, T. J. D’Arcy, D. J. Maxwell, W. Chiu, A. Milner, J. E. Saunders, and R. J. Sheppard. Volume estimation from multiplanar 2D ultrasound images using a remote electromagnetic position and orientation sensor. Ultrasound in Medicine and Biology, 22(5):561--572, 1996.

[7] D. F. Leotta, P. R. Detmer, and R. W. Martin. Performance of a miniature magnetic position sensor for three-dimensional ultrasound imaging. Ultrasound in Medicine and Biology, 24(4):597--609, 1997.

[8] T. R. Nelson and T. T. Elvins. Visualization of 3D ultrasound data. IEEE Computer Graphics and Applications, pages 50--57, November 1993. 
[9] D. L. King, D. L. King Jr., and M. Y. Shao. Evaluation of in vitro measurement accuracy of a three-dimensional ultrasound scanner. Journal of Ultrasound in Medicine, 10:77--82, 1991.

[10] R. Ohbuchi, D. Chen, and H. Fuchs. Incremental volume reconstruction and rendering for 3D ultrasound imaging. Proceedings of SPIE --- The International Society for Optical Engineering, pages 312--323, 1992.

[11] A. State, D. T. Chen, C. Tector, A. Brandt, H. Chen, R. Ohbuchi, M. Bajura, and H. Fuchs. Case study: Observing a volume rendered fetus within a pregnant patient. In Proc. IEEE Visualization, 1994, pages 364--368, 1994.

[12] J. W. Trobaugh, D. J. Trobaugh, and W. D. Richard. Three-dimensional imaging with stereotactic ultrasonography. Computerized Medical Imaging and Graphics, 18(5):315-- 323, 1994.

[13] Shattuck, D. P., Weishenker, M.D., Smith, S.W., and von Ramm, O.T. "Explososcan: A Parallel Processing Technique for High Speed Ultrasound Imaging with Linear Phased Arrays." JASA. 75(4): 1273-1282.

[14] Smith, S. W., Pavy, Jr., S.G., and von Ramm, O.T."High-Speed Ultrasound Volumetric Imaging System - Part I: Transducer Design and Beam Steering." IEEE Transaction on Ultrasonics, Ferro., and Freq. Control. 38(2): 100-108.

[15] P. R. Detmer, G. Bashein, T. Hodges, K. W. Beach, E. P. Filer, D. H. Burns, and D.E. Strandness Jr. 3D ultrasonic image feature localization based on magnetic scanhead tracking: in vitro calibration and validation. Ultrasound in Medicine and Biology, 20(9):923-936, 1994.

[16] D. F. Leotta, P. R. Detmer, and R. W. Martin. Performance of a miniature magnetic position sensor for three-dimensional ultrasound imaging. Ultrasound in Medicine and Biology, 24(4):597--609, 1997.

[17] R. W. Prager, R. Rohling, A. Gee, and L. Berman. Rapid calibration for 3-D freehand ultrasound. Ultrasound in Medicine and Biology, in press.

[18] Emad M. Boctor, Ashraf A. Saad, Prof A. M. Youseef, and Prof James Graham

"Heuristic Based Approach For Extracting Calibration Parameters of Ultrasound Equipment", presented in the ISCA International Conference, June 11-13, 1997, Boston, Massachusetts, USA.

[19] Ashraf A. Saad, Emad M. Boctor and Prof Abo Bakr Youssef(PhD/MD), "Statistical Based Automated Ultrasound Imaging Quality Control and Procedure", presented in the Fourth IEEE International Conference on Electronics, Circuits, and Systems ICECS'97, December 15-18, 1997, Cairo, Egypt.

[20] R. W. Prager, A. Gee, and L. Berman. STRADX: Real-Time Acquisition and Visualization of Free-Hand 3D Ultrasound CUED/F-INFENG/TR 319, Cambridge University Department of Engineering, April 1998.

[21] R. Ohbuchi, D. Chen, and H. Fuchs. Incremental volume reconstruction and rendering for 3D ultrasound imaging. Proceedings of SPIE - The International Society for Optical Engineering, 1808:312-323, 1992.

[22] G. Sakas, L-A. Schreyer, and M. Grimm. Preprocessing and volume rendering of 3D ultrasonic data. IEEE Computer Graphics and Applications, 15(4):47--54, July 1995. 